\title{
Association between real-time contrast-enhanced ultrasound characteristics and thyroid carcinoma size
}

\author{
ZHANG YUAN, JIANG QUAN, ZHANG YUNXIAO, CHEN JIAN and HE ZHU
}

Department of Ultrasound, Shanghai Pudong New Area People's Hospital, Pudong New Area, Shanghai 200001, P.R. China

Received November 13, 2014; Accepted March 24, 2015

DOI: $10.3892 / \mathrm{mco} .2015 .570$

\begin{abstract}
The aim of the present study was to discuss the various appearances of thyroid carcinomas on real-time contrast-enhanced ultrasound (CEUS) in association with tumor size. The appearances of 68 thyroid carcinomas confirmed by pathology were retrospectively analyzed. The lesions were divided into 3 groups by maximum diameter $<10,10-20$ and $>20 \mathrm{~mm}$, respectively. For each lesion, absolute enhancement beginning time, relative enhancement beginning time, homogeneity, with or without perfusion defect, enhancement order, enhancement intensity and enhancement margin type were evaluated by CEUS. The majority of thyroid carcinomas were enhanced later than the surrounding thyroid gland. The predominant enhancement pattern of all the 68 thyroid carcinomas in the 3 groups was mainly heterogeneous and concentric enhancement, and the lesions mostly showed less clear or poorly defined enhancement margins. There was no significant difference among the 3 groups $(\mathrm{P}>0.05)$. Thyroid carcinoma with diameters $<10$ and $10-20 \mathrm{~mm}$ exhibited low enhancement, while thyroid carcinomas with diameters $>20 \mathrm{~mm}$ showed high enhancement. With the increase of the lesion's maximum diameter, the probability of perfusion defect increased by $28.57,54.29$ and $75.00 \%$, respectively, and there was a significant difference among the 3 groups $(\mathrm{P}<0.05)$. CEUS characteristics are associated with tumor size to a certain extent, and can provide valuable information for clinical diagnosis.
\end{abstract}

\section{Introduction}

Thyroid nodules are commonly encountered lesions and have been observed in $50 \%$ of autopsied patients (1). The estimated annual incidence rate of $0.1 \%$ in the US suggests 300,000 newly diagnosed nodules as of 2005 (2). Although

Correspondence to: Dr Jiang Quan, Department of Ultrasound, Shanghai Pudong New Area People's Hospital, 490 Chuan Huan South Road, Pudong New Area, Shanghai 200001, P.R. China E-mail: 1079531821@qq.com

Key words: ultrasonography, microbubbles, thyroid carcinoma, tumor size only 1 of 20 clinically identified nodules is malignant (3), it is important to exclude the presence of a malignant thyroid lesion $(2,4)$.

Previous studies have demonstrated the feasibility of contrast-enhanced ultrasound (CEUS) for the differentiation of benign and malignant thyroid nodules (5). Nemec et al (6) reported that the complete CEUS data of 42 patients $(73.8 \%$ benign and $26.2 \%$ malignant nodules) revealed a significant difference in enhancement between benign and malignant nodules. Furthermore, CEUS demonstrated a sensitivity of $76.9 \%$, specificity of $84.8 \%$ and accuracy of $82.6 \%$. Quantitative analysis of CEUS using a microbubble contrast agent allows the differentiation of benign and malignant thyroid nodules and may potentially serve, in addition to grey-scale and Doppler ultrasound, as an adjunctive tool in the assessment of patients with thyroid nodules. Hornung et al (7) reported that CEUS represents a highly sensitive method for the detection of the microvascularization of thyroid carcinomas. Future studies should compare these findings to benign pathologies in order to establish CEUS as a standard diagnostic procedure in the preoperative evaluation of suspicious thyroid nodules. Zhang et al (8) reported that CEUS enhancement patterns were different in benign and malignant lesions. Ring enhancement was predictive of benign lesions, whereas heterogeneous enhancement was helpful for detecting malignant lesions.

The ultrasonic imaging characteristics of 68 patients with thyroid carcinoma proved by pathology were retrospectively analyzed in the present study. The correlation of real-time CEUS characteristic was mainly discussed in association with the lesion size.

\section{Materials and methods}

Study population. The study was approved by the Ethics Committee of Shanghai Pudong New Area People Hospital (Pudong, Shanghai, China), and patient consent was obtained. A total of 158 thyroid tumor cases were examined with CEUS in the hospital between January 2012 and August 2014. In total, 68 patients with thyroid carcinoma confirmed by pathology were recruited in the study. There were 31 male and 37 female patients with a mean age of $39.2 \pm 12.8$ years (range, 24-75 years) except thyroid diffuse diseases. All the 68 cases were solitary lesion with a mean diameter of $18 \pm 9 \mathrm{~mm}$ (max, 5-32 mm). Written informed consent was obtained from all the patients prior to the exam. All the cases were diagnosed 
by histological evaluation: Papillary carcinoma was diagnosed in 53 patients, follicular carcinoma in 11, papillary follicular carcinoma in 3 and medullary carcinoma in 1.

Ultrasound techniques. Siemens Sequoia 512 color Doppler ultrasound (Siemens, Bavaria, Germany) with the contrast pulse sequencing (CPS) image condition, probe model 15L8Ws and a frequency of $7 \mathrm{MHz}$ were used in the examination. All the patients were examined in the same conditions of mechanical index, depth, gain and time gain compensation. The contrast agent was SonoVue ${ }^{\circledR}$ (SonoVue, Bracco, Italy); 25 mg lyophilized powder with $5 \mathrm{ml}$ saline configured as a suspension, artificially agitated well.

Image analysis. Subjects lay supine on the examination table with their neck hyper-extended. All the examinations were performed by the same experienced operator. A two-dimensional high-frequency probe was used to observe location, size and Doppler flow signals of thyroid nodules. The largest section of the lesions was selected as the ultrasound imaging section, and when possible, the whole image and the surrounding area of the thyroid nodule were observed. The focus point was the place at the trailing edge of the lesion, and the gain was adjusted to reveal only the lesion boundaries. Subsequently, CPS was initiated by bolus injection through the elbow vein with $2.5 \mathrm{ml}$ contrast agent, washed with $5 \mathrm{ml}$ saline. When CPS started, the operator fixed the probe and asked the patient to avoid swallowing. The whole dynamic imaging process was stored on the machine's hard disk and ultrasound workstation for subsequent memorial processing and analysis. Real-time ultrasound contrast images were analyzed by the physicians with 5 years of ultrasound scanning experience. Each physician was blinded to the results of another physician. A senior physician analyzed the images when there were different opinions. The physicians discussed the images to provide the final agreement. In total there were 68 cases of thyroid cancer divided into 3 groups by the maximum diameter of the nodules; $<10,10-20$ and $>20 \mathrm{~mm}$. On observing the real-time ultrasound imaging process, the following data and analysis interpretation angiography characteristics were recorded: i) Absolute enhancement beginning time: From contrast agent bolus injected to contrast agent appearance; ii) relative enhancement beginning time: Contrast normal thyroid lesions began to increase early or late; iii) uniformity of enhancement: Homogeneous or heterogeneous enhancement (Fig. 1); iv) the existence of perfusion defects (Fig. 2); v) enhanced sequence: Concentric, eccentric or diffuse; vi) intensity of enhancement (compared to the surrounding normal thyroid): High, equal or low enhancement; vii) enhancement of lesion border: Clear, less clear or ill boundary (Fig. 3); For iii)-vii) characters were analyzed in contrast to the peak.

Statistical analysis. The SAS 8.0 statistical software (SAS Institute Inc., Cary, NC, USA) was used for data analysis. Measurement data are expressed as mean \pm standard deviation. Analysis of variance was used in the absolute enhancement beginning time of thyroid cancer. Enhancement characteristics were analyzed by the $\chi^{2}$ test, and $\mathrm{P}<0.05$ was considered to indicate a statistically significant difference.

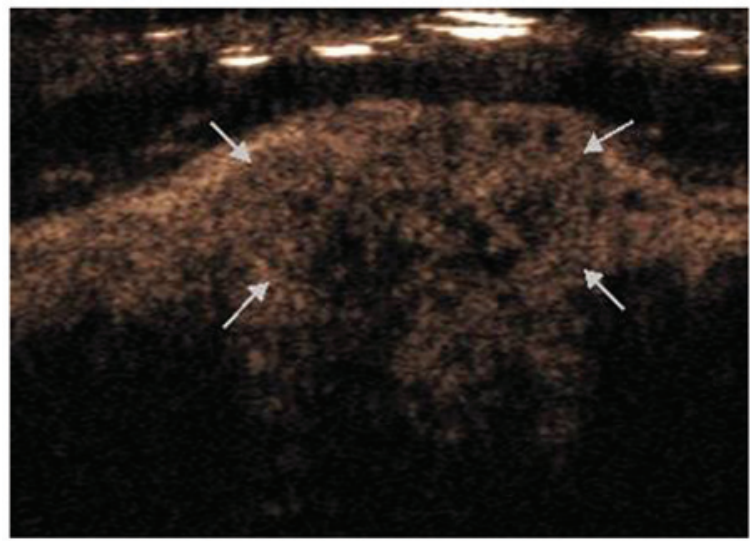

Figure 1. Contrast-enhanced ultrasound (CEUS) image of a 45-year-old male patient with a thyroid carcinoma of the left thyroid lobe demonstrating homogeneous or heterogeneous enhancement.

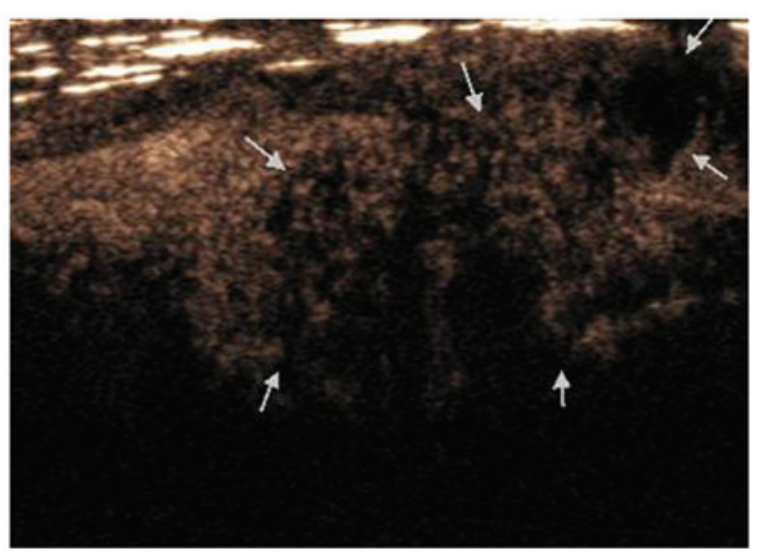

Figure 2. Contrast-enhanced ultrasound (CEUS) image of a 39-year-old female patient with a thyroid carcinoma of the right thyroid lobe demonstrating the existence of perfusion defects.

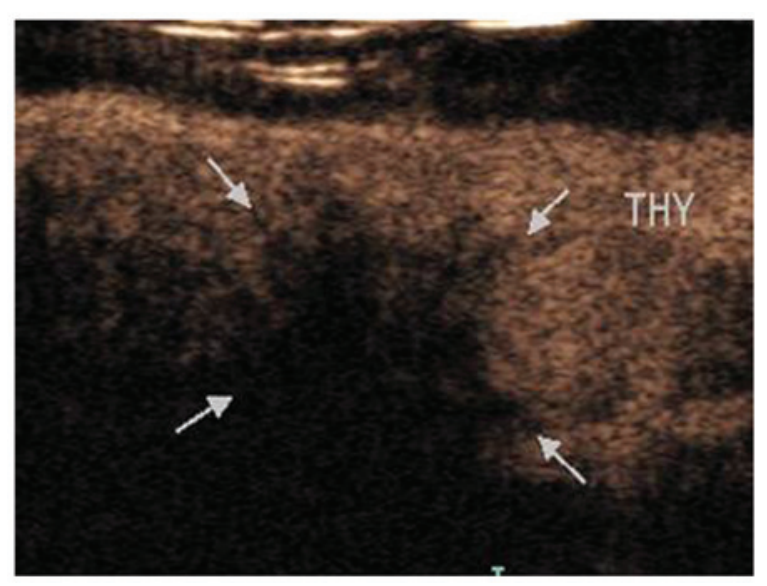

Figure 3. Contrast-enhanced ultrasound (CEUS) image of a 42-year-old female patient with a thyroid carcinoma of the right thyroid lobe demonstrating enhancement of the lesion border; less clear or ill boundary.

\section{Results}

Absolute enhancement beginning time of normal thyroid and thyroid cancer. The contrast agent began to increase 8-17 sec 
Table I. Association between relative enhancement beginning time and size of thyroid nodules.

\begin{tabular}{lrrrrr}
\hline $\begin{array}{l}\text { Relative } \\
\text { enhancement } \\
\text { beginning time }\end{array}$ & \multicolumn{3}{c}{ Tumor diameter, mm } & & \\
\cline { 2 - 4 } & $<10, \mathrm{n}$ & $10-20, \mathrm{n}$ & $>20, \mathrm{n}$ & $\chi^{2}$ & P-value \\
\hline Cases & 21 & 35 & 12 & & \\
Earlier & 2 & 3 & 1 & 0.0192 & 0.9905 \\
Equal & 6 & 9 & 3 & 0.0712 & 0.9650 \\
Later & 13 & 23 & 8 & 0.1079 & 0.9475 \\
\hline
\end{tabular}

${ }^{a}$ The P-values reflect the differences between enhancement characteristics in the different nodule size groups.

Table II. Association between enhancement characteristics and size of thyroid cancer.

\begin{tabular}{|c|c|c|c|c|c|}
\hline \multirow{2}{*}{$\begin{array}{l}\text { Enhancement } \\
\text { characteristics }\end{array}$} & \multicolumn{3}{|c|}{ Tumor diameter, mm } & \multirow[b]{2}{*}{$\chi^{2}$} & \multirow[b]{2}{*}{ P-value } \\
\hline & $<10, \mathrm{n}$ & $10-20, \mathrm{n}$ & $>20, \mathrm{n}$ & & \\
\hline Cases & 21 & 35 & 12 & & \\
\hline \multicolumn{6}{|l|}{$\begin{array}{l}\text { Uniformity of } \\
\text { enchancement }\end{array}$} \\
\hline Homogeneous & 3 & 4 & 2 & 0.0099 & 0.9209 \\
\hline Heterogeneous & 18 & 31 & 10 & & \\
\hline Perfusion defects & 6 & 19 & 9 & 6.9695 & 0.0083 \\
\hline \multicolumn{6}{|l|}{ Enhanced sequence } \\
\hline Concentric & 13 & 23 & 8 & 0.0576 & 0.9716 \\
\hline Eccentric & 5 & 4 & 1 & & \\
\hline Diffuse & 3 & 8 & 3 & & \\
\hline \multicolumn{6}{|l|}{$\begin{array}{l}\text { Intensity of } \\
\text { enhancement }\end{array}$} \\
\hline High & 1 & 3 & 7 & 14.4743 & 0.0001 \\
\hline Equal & 3 & 5 & 3 & & \\
\hline Low & 17 & 27 & 2 & & \\
\hline \multicolumn{6}{|l|}{$\begin{array}{l}\text { Enhancement of } \\
\text { lesion border }\end{array}$} \\
\hline Clear & 1 & 4 & 2 & 0.7126 & 0.3986 \\
\hline Less clear & 5 & 11 & 2 & & \\
\hline Ill & 15 & 20 & 8 & & \\
\hline
\end{tabular}

${ }^{a}$ The P-values reflect the differences between enhancement characteristics in the different nodule size groups.

after injection in the normal thyroid tissue, with an average of $12.28 \pm 1.16 \mathrm{sec}$. In total, 68 cases of thyroid cancer were enhanced. The absolute enhancement beginning time for the diameter of carcinoma being $<10,10-20$ and $>20 \mathrm{~mm}$ was $15.21 \pm 3.62,14.88 \pm 3.45$ and $14.59 \pm 3.17 \mathrm{sec}$, respectively. There was no statistical significance among the 3 groups $(\mathrm{P}>0.05)$.

Association between relative enhancement beginning time and size of thyroid cancer. The 3 groups of thyroid cancer enhanced later than the surrounding normal thyroid (Table I). There was no statistical significance among the 3 groups $(\mathrm{P}>0.05)$.

Association between enhancement characteristics and size of thyroid cancer. All the 3 groups of thyroid cancer showed heterogeneous and concentric enhancement (Table II). There was no statistical significance among the 3 groups $(\mathrm{P}>0.05)$. In lesions with diameters $<10 \mathrm{~mm}$ or between $10-20 \mathrm{~mm}$, insignificant enhancement was usually observed, while in lesions with diameter $>20 \mathrm{~mm}$, hyper-enhancement was usually observed. The difference was statistically significant $(\mathrm{P}<0.05)$. With the increase of diameter, the perfusion defects of nodules increased by $28.57,54.29$ and $75.00 \%$, respectively. The difference was statistically significant $(\mathrm{P}<0.05)$.

\section{Discussion}

A tumor is a type of typical vascular-dependent lesion. Small blood vessels with diameters $<40 \mu \mathrm{m}$ can be detected by CEUS. Previously, clear results have been achieved in studying tumor vascular perfusion features by CEUS for focal liver lesions, but the study of its application in thyroid nodules was only preliminary (9-12). In the present study, real-time CEUS images for 68 cases of patients with thyroid cancer were retrospectively analyzed with the main purpose of exploring the association between characteristics of thyroid carcinomas in real-time CEUS and tumor sizes.

In the present study, the absolute enhancement beginning time for different lesions were not statistically significant, as the enhancement beginning time following injection of the contrast agent could be affected by various factors, such as differences in time of contrast agent bolus injection among different operators and difference in intimate circulation of the contrast agent among different individuals. Therefore, relative enhancement beginning time was used to evaluate the enhancement time of different lesions. The majority of lesions enhanced later than the surrounding thyroid gland and the differences in features of enhancement for lesions among different groups were not statistically significant. The predominant enhancement pattern in different groups was heterogeneous concentric enhancement, and the majority of lesions showed less clear or poorly defined margins following enhancement. The study by Zheng et al (13) reported that 35 thyroid carcinomas presented three enhancement patterns with CEUS. These were type I: 23 lesions enhanced in a pattern of ring with centripetal fill-in, however, the central part had no contrast agent filling; type II: 5 lesions enhanced regularly and homogeneously; and type III: 7 lesions enhanced irregularly and homogeneously.

We believe that the above enhancement features are in accordance with the pathological features. Vascular pathological anatomy for thyroid cancer is complicated. Generally, neovascular could be divided into surrounding and central area, which showed different vascular distribution characteristics. Blood vessels in the surrounding area are relatively concentrated and the tumor usually grows infiltratively outward, which leads to the formation of less clear or poorly defined margins following enhancement; while blood vessels in the central area are relatively less concentrated. Therefore, the difference in the abundance of blood vessels between the surrounding and central area are the main reason for concentric enhancement. 
For the growth heterogeneity and neovascular damage caused by malignant infiltration, thyroid cancer is always combined with fibrosis and hyalinization degeneration. The original vascular networks would be damaged, which results in coexistence of abundant blood supply in certain areas and inabundant blood supply in other areas. Existence of various arteriovenous fistulas enhanced the imbalance of vascular networks (14). All the abovementioned reasons contribute to the heterogeneous enhancement in CEUS for thyroid cancer.

As indicated in the present study, the incidence of perfusion defect within lesions increased with the increase of lesion diameter, which is consistent with the growth feature of malignant tumor. A previous study reported that during the growth of the malignant tumor, the doubling time for vascular endothelial cells and tumor cells is different, which indicates that the tumor growth speed is much higher than the formation speed of microvessels (15). The increase of the microvessel number is relatively slower and the incidence is more evident with the growth of the tumor. With the growth of the tumor, the blood supply in the lesion is poorer, so the incidence of part or complete defect is higher, and this results in a higher rate of local perfusion defects.

Bartolotta et al (5) identified that the enhancement pattern of thyroid nodules in CEUS was closely associated with nodule size, which was indicated as an inabundant blood supply in CEUS for malignant lesions $<10 \mathrm{~mm}$, few nodular enhancements for 10-20 mm lesion and diffuse enhancement for lesions $>20 \mathrm{~mm}$. With a larger sample, the enhancement features in CEUS for groups with different lesion sizes were analyzed in this study. The results indicate that, in groups with a lesion diameter $<10 \mathrm{~mm}$ and between 10-20 mm, CEUS mainly showed a not significant enhancement, but mainly hyper-enhancement in the group with a lesion size $>20 \mathrm{~mm}$. The following may be the reasons for this: Growth of the tumor consists of two stages, from the slow-growing stage without blood vessels (pre-vascular phase) to fast-growing stage with blood vessels (vascular phase). Without or with fewer blood vessels, the enhancement observed in CEUS for relatively smaller tumors was mainly insignificant enhancement. When the tumor grows quickly, various new blood vessels form under the introduction of multiple angiogenic factors to meet the requirements of fast growth. With complicated vascular network, hyper-enhancement was usually observed in relatively large thyroid cancer lesions.

In conclusion, there is a certain correlation between enhancement features in CEUS for thyroid cancer and lesion size. In lesions with diameters $<10 \mathrm{~mm}$ or between $10-20 \mathrm{~mm}$, insignificant enhancement was usually observed, while in lesions with diameters $>20 \mathrm{~mm}$, hyper-enhancement was usually observed. The incidence of perfusion defects within the lesion increases with larger lesion diameters. Real-time CEUS can provide valuable information for clinical diagnosis.

\section{Acknowledgements}

The study was supported by grants from Pudong New Area Health Plan Board of Health Science and Technology Project in Shanghai (no. PW2014A-23), Pudong New Area leading talents training plan (no. PWR12012-02), Shanghai
Health Bureau research projects (no. 20134059) and Shanghai Pudong Science and Technology Innovation Fund (no. PKJ2012-Y56).

\section{References}

1. Mortensen JD, Woolner LB and Bennett WA: Gross and microscopic findings in clinically normal thyroid glands. J Clin Endocrinol Metab 15: 1270-1280, 1995.

2. Gharib H, Papini E, Paschke R, Duick DS, Valcavi R, Hegedüs L and Vitti P: American Association of Clinical Endocrinologists, Associazione Medici Endocrinologi and European Thyroid Association medical guidelines for clinical practice for the diagnosis and management of thyroid nodules: Executive summary of recommendations. J Endocrinol Invest 33: 51-56, 2010.

3. Hegedus L: Clinical practice. The thyroid nodule. N Engl J Med 351: 1764-1771, 2004.

4. Frates MC, Benson CB, Charboneau JW, Cibas ES, Clark OH, Coleman BG, Cronan JJ, Doubilet PM, Evans DB, Goellner JR, Hay ID, Hertzberg BS, Intenzo CM, Jeffrey RB, Langer JE, Larsen PR, Mandel SJ, Middleton WD, Reading CC, Sherman SI and Tessler FN: Management of thyroid nodules detected at US: Society of radiologists in ultrasound consensus conference statement. Radiology 237: 794-800, 2005

5. Bartolotta TV, Midiri M, Galia M, Runza G, Attard M, Savoia G, Lagalla R and Cardinale AE: Qualitative and quantitative evaluation of solitary thyroid nodules with contrast-enhanced ultrasound: initial results. Eur Radiol 16: 2234-2241, 2006.

6. Nemec U, Nemec SF, Novotny C, Weber M, Czerny C and Krestan CR: Quantitative evaluation of contrast-enhanced ultrasound after intravenous administration of a microbubble contrast agent for differentiation of benign and malignant thyroid nodules: assessment of diagnostic accuracy. Eur Radiol 22: 1357-1365, 2012.

7. Hornung M, Jung EM, Georgieva M, Schlitt HJ, Stroszczynski C and Agha A: Detection of microvascularization of thyroid carcinomas using linear high resolution contrast-enhanced ultrasonography (CEUS). Clin Hemorheol Microcirc 52: 197-203, 2012 .

8. Zhang B, Jiang YX, Liu JB, Yang M, Dai Q, Zhu QL and Gao P: Utility of contrast-enhanced ultrasound for evaluation of thyroid nodules. Thyroid 20: 51-57, 2010.

9. Friedrich-Rust M, Sperber A, Holzer K, Diener J, Grünwald F, Badenhoop K, Weber S, Kriener S, Herrmann E, Bechstein WO, Zeuzem $S$ and Bojunga J: Real-time elastography and contrast-enhanced ultrasound for the assessment of thyroid nodules. Exp Clin Endocrinol Diabetes 118: 602-609, 2010.

10. Xu HX: Contrast-enhanced ultrasound: The evolving applications. World J Radiol 1: 15-24, 2009.

11. Agha A, Hornung M, Rennert J, Uller W, Lighvani H, Schlitt HJ and Jung EM: Contrast-enhanced ultrasonography for localization of pathologic glands in patients with primary hyperparathyroidism. Surgery 151: 580-586, 2012.

12. Giusti M, Orlandi D, Melle G, Massa B, Silvestri E, Minuto F and Turtulici G: Is there a real diagnostic impact of elastosonography and contrast-enhanced ultrasonography in the management of thyroid nodules? J Zhejiang Univ Sci B 14: 195-206, 2013 (In Chinese).

13. Zheng XJ, Zhang YK, Zhao CY, Liang JR, LE HB, Jiang JF, Wang H, Zou SD and Chen YF: Enhancement pattern of thyroid carcinoma with contrast-enhanced ultrasound. Zhonghua YiXue Za Zhi 90: 42-45, 2010 (In Chinese).

14. Averkious M, Powers J, Skyba D, Bruce M and Jensen S: Ultrasound contrast imaging research. ultrasound Q 19: 27-37, 2003.

15. Jain RK: Normalizing tumor vasculature with anti-angiogenic therapy: A new paradigm for combination therapy. Nat Med 7: 987-989, 2001. 